\title{
Long periods with uninterrupted muscle activity related to neck and shoulder pain
}

\author{
Therese N Hanvold, Morten Wærsted and Kaj Bo Veiersted ${ }^{1}$ \\ Department of Work Psychology and Physiology, National Institute of Occupational Health, P.O. Box 8149, \\ N-0033 Oslo, Norway
}

\begin{abstract}
The aim was to analyze the relationship between periods with uninterrupted neck muscle activity for $\geq 4$ min and neck and shoulder pain. The trapezius muscle activity was recorded bilaterally on 40 young workers and students during a full shift. Neck and shoulder pain, mechanical work load and decision control were reported at the same time as the muscle activity recording and 6 months later. A dose-response relationship was found between uninterrupted muscle activity and neck and shoulder pain, with a ten-fold higher risk for the group with more than half, compared to less than a third, of the shift with uninterrupted muscle activity. Self-reported mechanical work load showed a small but protective effect related to pain. Gender and decision control did not emerge as important risk factors in this model. In conclusion, this study indicates that work or other exposures that contains long periods with uninterrupted neck muscle activity of 4 min duration or longer should be minimized to reduce risk of neck and shoulder pain.
\end{abstract}

Keywords: prospective, motor control, sustained muscle activity, neck and shoulder pain

\section{Introduction}

Sustained low-level force muscle contractions have been suggested as a risk factor for work-related neck pain $[3,10]$. Insufficient muscle rest [6] or long total time with neck muscle activity above $2 \%$ of maximal contractions ("bursts", [9]) are possible characteristics of muscle activity pattern related to pain. However, these measures do not take into account the duration of periods with sustained muscle contraction. Long periods with uninterrupted muscle fiber activation may constitute a risk for pain development [2]. An index of different mechanical work load exposures [4] and psychosocial factors as decision control at work [5] have also been related to neck and shoulder pain.

Increased neck pain reports has been found among forest machine operators with a muscle activity pattern showing frequent uninterrupted periods of longer than 8 minutes duration [13]. The study of middleaged experienced forest machine operators is the only known that relates duration of uninterrupted muscle activity periods to the development of neck pain after $1 / 2$ to 1 year.

The aim of the present paper is therefore to examine if long periods with uninterrupted neck muscle activation is a risk factor for neck pain also in young workers.

This proceeding-paper is based in parts on a full size manuscript that at present (fall 2011) is in a process to be published in a peer-review journal (TN Hanvold and co-workers: A $41 / 2$ year prospective study on sustained trapezius muscle activity at work and neck and shoulder pain among young adults.)

\section{Methods}

This study is based on results from a prospective cohort study performed from 2002 to 2009 of 420 young subjects followed from the second year of technical school and into a working or studying career. The neck muscle activity during work or study was measured in 2007 for a subgroup. This paper reports data on risk factors and neck pain at that time

\footnotetext{
${ }^{1}$ Corresponding author. E-mail: bo.veiersted@stami.no, tel. no. +4723195375, fax no. +4723195204 .
} 
(time " 0 ") and the following $1 / 2$ a year for this subgroup.

\subsection{Subjects}

Forty subjects constituted this subgroup, consisting of hairdressers (15 females), electricians (14 males), various jobs ( 3 females, 3 males) and students (5 females). At basis (2002) the future subgroup included in this study had a significant higher frequency of smokers compared to the rest of the cohort, in 2007 no difference was found. No difference was found between the two groups reporting of physical activity or neck and shoulder pain, neither for the 2002 nor for the 2007 examination. The median age was 22 years (range 21-25) and median body mass index was 21.7 (range 17.5-28.2).

The Regional Committee for Medical Research Ethics approved the study and an informed written consent form was obtained from all participants.

\subsection{Electromyography}

Surface electromyography (EMG) of upper trapezius muscle was recorded bilaterally during a fullshift (median 318 minutes (range 153-429) at time "0"). Bipolar electrodes with $6 \mathrm{~mm}$ gel were placed in standardized positions [11] and normalization according to standardized procedures $[1,11]$. The RMS length was $1.64 \mathrm{~s}$ and discrimination level for muscle activity was $0.5 \%$ of $\mathrm{EMG}_{\max }$ [13]. The fraction (relative time) of total shift with uninterrupted muscle activity for 4 min periods or longer, was analyzed as a mean of both sides. No data were rejected since the quality assessment did not reveal any movement artifacts or electromagnetic inference. The whole group was divided in three subgroups depending on the fraction of uninterrupted muscle activity $\geq 4 \mathrm{~min}$ duration: $0-30 \%, 31-50 \%$ and $50-100 \%$ of the fullshift recording.

\subsection{Neck and shoulder pain}

The outcome variable of neck and shoulder pain the preceding month included both intensity (not troubled, little troubled, quite troubled and very troubled) and duration (1-5 days, 6-10 days, 11-14 days and 15-28 days). On the basis of this reporting a pain index was calculated by multiplying pain intensity (0-3) and duration (1-4), giving a range from 0 to
12 [8]. Data from time of the EMG recording (time " 0 ") and 6 months later were used in this paper.

\subsection{Self-reported risk factors}

Except for the muscle activity pattern measured by EMG (see above), three other potential risk factors were included in this paper. One factor was gender. Twelve questions were used to form an index (score 0-24) of the work-related mechanical exposure [4]. The questions included assessment of repetitive movements, manual material handling, awkward postures of neck and low back (index named MI2 in [4]). Two questions were used in an index (score 0-4) to measure the decision control over work intensity; "can you set your own work pace?" and "can you determine the lengths of your own breaks?". These questions were selected from the General Nordic Questionnaire for Psychological and Social factors at Work $\left(\mathrm{QPS}_{\text {Nordic }}\right)$ [7].

\subsection{Statistical analysis}

The multivariate analysis was performed by generalized estimating equation (GEE). GEE-analysis takes into account the correlation between the repeated measurements within the individual. Due to over dispersion in the discrete outcome variable, a negative binomial GEE-analysis with an exchangeable correlation structure was used. For the effect estimates, incidence rate ratio (IRR), with corresponding $95 \%$ confidence intervals were reported.

\section{Results}

\subsection{Description of potential risk factors}

Table 1 shows the distribution of the potential risk factors used in the model (see later). The median of self-reported mechanical work load was equal in the three groups, and decision control showed a decreasing tendency. 
Table 1

Risk factors for the three fraction groups. Numbers are shown for gender. Median and range (in brackets) are shown for the other variables.

Fraction of uninterrupted muscle activity periods $\geq 4$ min duration

Low Moderate High

\begin{tabular}{lccc} 
Risk factors: & $0-30 \%$ & $31-50 \%$ & $51-100 \%$ \\
\hline Muscle activity, & 20 & 40 & 66
\end{tabular}

\begin{tabular}{lccc}
$(\%)$ & $(2-30)$ & $(32-50)$ & $(52-91)$ \\
\hline Gender $(\mathrm{N})$, male & 8 & 5 & 4
\end{tabular}

\begin{tabular}{lccc}
\multicolumn{1}{c}{ female } & 6 & 9 & 8 \\
\hline Mechanical & 10 & 11 & 11 \\
workload (score) & $(1-18)$ & $(3-19)$ & $(5-18)$ \\
\hline Decision control & 2.5 & 2.5 & 2 \\
(score) & $(1.5-4)$ & $(0-4)$ & $(0.5-3)$ \\
\hline
\end{tabular}

\subsection{Neck and shoulder pain}

The overall level of neck and shoulder pain was rather low. The mean level increased from just below 2 at time " 0 " to just below 4 (of a maximum of 12) after 6 months for the high fraction group (Figure 1).

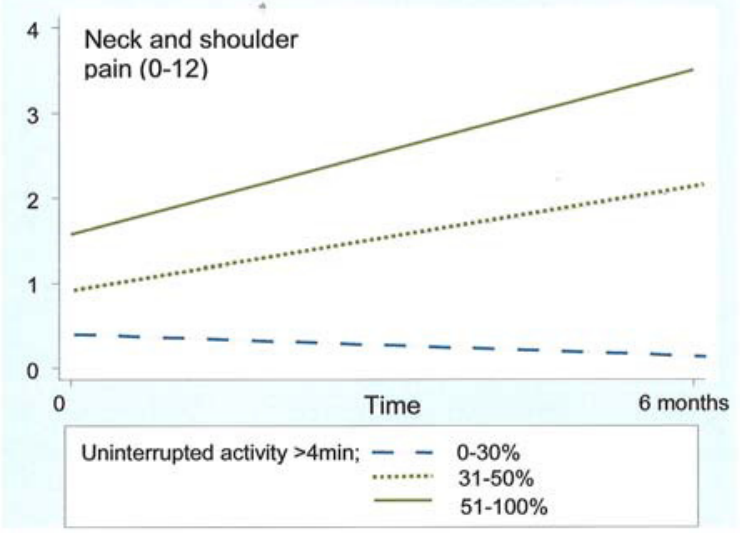

Figure 1. Reports of neck and shoulder pain divided into the three groups of different fraction of uninterrupted neck muscle activity.

\subsection{Multivariate analysis}

The multivariate analysis (Table 2) showed a highly significant effect of increased fraction of uninterrupted muscle activity on reports of neck and shoulder pain. A small and paradoxical protective effect was shown for self-reported mechanical work load. Gender and decision control did not contribute to increased risk for the outcome in this model.
Table 2.

Multivariate analyzes of risk factors for neck and shoulder pain at time " 0 " and 6 months later. Model based on a Generalized estimating equation (GEE).

\begin{tabular}{lccc} 
& \multicolumn{3}{c}{ Incidence rate ratio } \\
\cline { 2 - 4 } Risk factors: & IRR & $95 \%$ CI & p-value \\
\hline Act $\geq 4$ min*, $0-30 \%$ & 1.00 & & \\
$31-50 \%$ & 6.38 & $2.01,20.24$ & 0.002 \\
$51-100 \%$ & 10.03 & $3.35,29.99$ & $<0.001$ \\
\hline $\begin{array}{l}\text { Gender } \\
\text { (male highest) }\end{array}$ & -1.07 & $-2.92,2.54$ & 0.891 \\
\hline $\begin{array}{l}\text { Mechanical } \\
\text { workload }\end{array}$ & -1.06 & $-1.11,-1.01$ & 0.011 \\
\hline $\begin{array}{l}\text { Decision control } \\
\text { *Uninterrupted muscle activity periods }>0.5 \% \mathrm{EMG}_{\max } \text { for } 4 \mathrm{~min}\end{array}$ \\
$\begin{array}{l}\text { or longer. } \\
-1.01\end{array}$ & $-1.20,1.15$ & 0.814 \\
\end{tabular}

\section{Discussion}

Some indications exist in our data that long periods with uninterrupted muscle activity $\geq 4 \mathrm{~min}$ constitute a risk factor for neck and shoulder pain. This is in accordance with the conclusions of a recent thesis [10]. The dose-response relationship strengthens the found association. However, the confidence intervals are wide for moderate and high fractions of uninterrupted muscle activity. Self-reported mechanical work load showed a small but surprisingly protective effect on neck and shoulder pain. This tendency was also found in a larger subsample from the same cohort [12], which suggests that this index variable do not cover risk factors of mechanical work load related to neck and shoulder pain in this group of young adults. Gender and psychosocial factors, as decision control, seem of less importance in the used model.

The level of neck and shoulder pain is rather low in this study of young adults. The risk found is therefore associated to the incidence of light symptoms. At least two weaknesses of this study must be stressed. One is the low number of subjects and the other is that the analyses only consider duration of time with uninterrupted muscle activity and do not take into account restitution time. The relation between muscle activity and rest may be an important aspect of beneficial muscle activity pattern.

In conclusion, this study indicates that work or other exposures that contains long periods with uninterrupted neck muscle activity of 4 min duration or 
longer should be minimized to reduce risk of neck and shoulder pain.

\section{Acknowledgements}

We are grateful for the collaboration from the participants and the employers making it possible to perform the data collection. We are also grateful for the assistance with the technical measurements by Ada Ingvaldsen, Birgitta J. Robertsson, Jorid T. Stuenæs and Einar Jebens. This study was, in part, supported by the Swedish Council for Work Life and Social Research, AFA Insurance, the Medical Faculty of Lund University, the county councils of southern Sweden and grants from the Confederation of Norwegian Enterprises (NHO).

\section{References}

[1] G.-Å. Hansson, P. Asterland, and S. Skerfing. Acquisition and analysis of whole-day electromyographic field recordings. In: SENIAM, European Applications on Surface Electromyography. Proceeding of the second general SENIAM workshop, Stockholm, Sweden, June 1997. H. J. Hermens, G. Hägg, \& B. Freriks, eds. Roessing Research and Development, The Netherlands, 1997, pp. 19-27.

[2] G.M. Hägg. Static work loads and occupational myalgia - a new explanation model. In: Anderson PA, Hobart DJ, Danoff JV, editors. Electromyographical kinesiology. Amsterdam: Elsevier Science Publishers B.V., 1991. pp. 141-144.

[3] G. Sjøgaard, U. Lundberg, R. Kadefors. The role of muscle activity and mental load in the development of pain and degenerative processes at the muscle cell level during computer work. Eur J Appl Physiol 83 (2000), 99-105.

[4] I. Balogh, P. Ørbæk, J.Winkel, et al., Questionnaire-based mechanical exposure indices for large population studies-- reliability, internal consistency and predictive validity. Scand J Work Environ Health 27 (2001), 30-40.

[5] J.-O. Christensen and S. Knardahl, Work and neck pain: A prospective study of psychological, social, and mechanical risk factors. Pain 151 (2010), 162-173.

[6] K.B. Veiersted, RH Westgaard, P. Andersen. Electromyographic evaluation of muscular work pattern as a predictor of trapezius myalgia. Scand J Work Environ Health 19 (1993), 284-90.

[7] M. Dallner, A.-L. Elo, F. Gamberale, et.al. Validation of the General Nordic Questionnaire (QPSnordic) for psychological and social factors at work. Copenhagen: Nordic Council of Ministers, 2000.

[8] O.A. Steingrímsdóttir, N.K. Vøllestad, C. Røe, et al.. Variation in reporting of pain and other subjective health complaints in a working population and limitations of single sample measurements. Pain 110 (2004), 130-9.

[9] P.J. Mork, R.H. Westgaard. Low-amplitude trapezius activity in work and leisure and the relation to shoulder and neck pain. J Appl Physiol 100 (2006), 1142-1149.

[10]P. Madeleine, On functional motor adaptations: from the quantification of motor strategies to the prevention of musculoskeletal disorders in the neck-shoulder region (thesis). Acta Physiol (Oxf) 199 Suppl 679, 2010.

[11] S.E. Mathiassen, J. Winkel, and G.M. Hägg. Normalization of surface EMG amplitude from the upper trapezius muscle in ergonomic studies - a review. Journal of Electromyography and Kinesiology 5,(1995), 197-226.

[12] T.N. Hanvold, K.B. Veiersted, M. Wærsted. A prospective study of neck, shoulder and upper back pain among technical school students entering working life. Journal of Adolescent Health 46 (2010), 488-494.

[13]T. Østensvik, K.B. Veiersted, P. Nilsen. Association between numbers of long periods with sustained low-level trapezius muscle activity and neck pain. Ergonomics 52 (2009), 15561567. 